\title{
COVID-19-Impfung: Haftung und Patientenrechte
}

\author{
Ursina Pally Hofmann
}

Dr. iur., Rechtsanwältin, Generalsekretärin und Leiterin Rechtsdienst FMH

\section{Haftungsgrundlagen}

\section{Übersicht}

Die geltende Rechtsordnung sieht im Zusammenhang mit einer Impfung grundsätzlich zwei primäre Haftungssubjekte vor. Das ist zum einen der Hersteller des Impfstoffs, zum anderen der impfende Arzt. Ebenso hat der Arzt zur Umsetzung des nationalen Impfplans beizutragen.

Die folgenden Ausführungen sollen Ihnen aufzeigen, wie die Haftungsfragen in derartigen Fällen beantwortet werden und welches Ihre Pflichten sind.

Bei behördlich angeordneten und empfohlenen Impfungen kommt eine subsidiäre Haftung für Impfschäden in Frage, wenn ein Ersatz für Impfschäden nicht bei den primär haftenden Personen geltend gemacht werden kann.

\section{Produktehaftung}

Das Produktehaftpflichtgesetz [1] sieht die Haftung des Herstellers vor, wenn durch dessen fehlerhaftes Produkt ein Schaden, z.B. die Tötung oder Verletzung eines Menschen, gesetzt wird. Die Herstellerin ist jene juristische oder natürliche - Person, welche das Endprodukt, einen Grundstoff oder ein Teilprodukt

\section{Die Sicherheitserwartungen richten sich nach den Fachinformationen, nicht nach den Patien- teninformationen im Beipackzettel.}

hergestellt hat. Allenfalls können auch Lieferanten oder Personen, die sich als Herstellerin ausgeben, haftbar gemacht werden [2]. Als Produkte gelten unter anderem Medikamente [3].

Fehlerhaft ist ein Produkt, das nicht die Sicherheit bietet, welche unter Berücksichtigung aller Umstände erwartet werden kann. Die Umstände, welche bei dieser Beurteilung eine Rolle spielen, sind die Art und Weise der Produktepräsentation, der Gebrauch, mit dem man vernünftigerweise rechnen kann, und der Zeitpunkt, in welchem es in Verkehr gebracht wurde [4].
Die Sicherheitserwartungen bei rezeptpflichtigen Medikamenten - wozu auch Impfungen gehören - richten sich nach den Fachinformationen, welche der Arzt kennen und mit der Patientin diskutieren muss, und nicht nach der Patienteninformation im Beipackzettel [5]. Eine Produktehaftung kommt nicht in Frage, wenn die Patienteninformation keine oder nur ungenügende Warnhinweise enthält, die Fachinformation hingegen vollständig ist.

Die Haftung verlagert sich deshalb auf den impfenden Arzt, welcher die Patientin nicht genügend, das heisst gemäss den diesbezüglichen Fachinformationen, aufklärt.

\section{Vertragliche Haftung - Staatshaftung}

Grundsätzlich haftet die Ärztin für eine unsorgfältige Behandlung oder mangelhafte Aufklärung gemäss privatvertraglichen Bestimmungen, wobei die jeweilige Versicherungspolice den Schaden deckt, sofern die Haftungsvoraussetzungen erfüllt sind [6].

Bei behördlich angeordneten Impfungen handelt die Ärztin als staatliches Vollzugsorgan. Sofern es die kantonalen Haftungs- und Verantwortlichkeitsgesetze vorsehen, kommt in diesen Fällen die Staatshaftung zum Tragen.

Unklar ist die Lage betreffend Haftungsgrundlage bei lediglich empfohlenen Impfungen, da sie freiwillig sind.

Die unterschiedlichen Haftungsgrundlagen spielen betreffend die Pflichten der impfenden Ärztin keine Rolle. In beiden Fällen ist sorgfältig zu behandeln und die Patientin genügend aufzuklären. Der wesentliche Unterschied besteht darin, dass der Schaden in einem Fall über die Haftpflichtpolice des fraglichen Arztes und im anderen Fall über jene des betreffenden Gemeinwesens bezahlt würde.

\section{Haftung für rechtswidrige Impfobligatorien} Besteht eine erhebliche Gefahr für besonders exponierte Personen und für Personen, die bestimmte Tätigkeiten ausüben, können die Kantone Impfungen für obligatorisch erklären [7]. Die Erheblichkeit der Gefahr wird anhand verschiedener Kriterien festgelegt, wie 
z.B. des Schweregrads der möglichen Erkrankung, der zu erwartenden Wirksamkeit des Obligatoriums und der epidemiologischen Situation [8].

Das Obligatorium muss zeitlich befristet sein, und die Impfung darf nicht mittels physischen Zwangs erfolgen [9]. Deshalb ist auch in den Fällen, in welchen ein Impfobligatorium besteht, jede Patientin vor einer Impfung über die Impfung und damit verbundene Folgen aufzuklären. Sie darf nur geimpft werden, wenn sie

\section{Unabhängig von den Haftungsgrundlagen ist der Patient immer sorgfältig zu behandeln und genügend aufzuklären.}

nach erfolgter Aufklärung ihre Einwilligung dazu gibt. Es darf also auch im Falle einer obligatorischen Impfung niemand gegen seinen Willen geimpft werden. Werden diese Grundsätze bei der Anordnung eines Impfobligatoriums verletzt, und sind die übrigen Haftungsvoraussetzungen - Schaden und Kausalzusammenhang zwischen Obligatorium und Schaden erfüllt, kommt es zu einer Staatshaftung gemäss kantonalen Haftungsgesetzen.

\section{Haftung für unsorgfältige Anwendung eines Impfstoffes}

Ärztinnen sind verpflichtet, im Rahmen ihrer Tätigkeit zur Umsetzung des nationalen Impfplans beizutragen. Sie müssen die von den Impfempfehlungen betroffenen Personen über den nationalen Impfplan informieren. Dazu gehört auch die Information betreffend die Übertragungs- und Krankheitsrisiken für Personen, die nicht geimpft sind. Weiter hat die Ärztin die offiziellen Informationsmittel des Bundes und der Kantone den von den Empfehlungen betroffenen Personen zur Verfügung zu stellen [10].

\section{Subsidiäre Haftung des Bundes nach Epidemiengesetz}

Das Epidemiengesetz sieht vor, dass ein Anspruch auf Entschädigung für Impffolgeschäden bejaht werden kann, wenn die Impfung behördlich angeordnet oder empfohlen worden ist. Eine Entschädigung wird allerdings erst dann bezahlt, wenn der Schaden mit zumutbaren Bemühungen nicht anderweitig gedeckt werden konnte [11].

Wird ein Patient folglich durch die fehlerhafte Aufklärung, durch eine unsorgfältige Impfung oder einen fehlerhaften Impfstoff geschädigt, kommt eine Entschädigung nach Epidemiengesetz nur in Frage, wenn die Ansprüche des Patienten nicht von der Versicherung des Arztes bzw. des Herstellers des Impfstoffs gedeckt sind oder diese Ansprüche abgelehnt werden.

\section{Pflichten des impfenden Arztes}

\section{Sorgfalt}

Eine Haftung wegen Impfschäden kann begründet werden, wenn nachgewiesen wird, dass der Arzt die Impfung unsorgfältig vorgenommen hat, und diese Unsorgfalt die Ursache für den Schaden ist.

Eine Verletzung der Sorgfaltspflicht liegt etwa dann vor, wenn die Impfeignung einer Person nicht abgeklärt wird, nicht zugelassene oder ungeeignete Impfstoffe verwendet, die Hygienestandards nicht beachtet werden oder der Impfstoff in einer falschen Dosis appliziert wird. Ebenso kann die falsche Lagerung des Impfstoffs zu einer Haftung führen, sofern sie einen Gesundheitsschaden verursacht.

\section{Aufklärung}

Der weit überwiegende Teil allfälliger Haftungsforderungen dürfte wohl mit einer fehlenden oder unvollständigen Aufklärung begründet werden, denn die Behandlung "Impfen» ist aus rein technischer Sicht im Vergleich $\mathrm{zu}$ anderen medizinischen Behandlungen nicht sehr anspruchsvoll, weshalb die Sorgfaltspflichten mehrheitlich problemlos eingehalten werden können. Deshalb ist auch bei der Impfung mit einem COVID-19-Impfstoff besonderes Augenmerk auf die rechtsgenügende Aufklärung zu legen.

Grundsätzlich muss der Patient über die Art und Risiken der in Aussicht gestellten Impfung so aufgeklärt werden, dass er in Kenntnis der Sachlage einwilligen kann [12].

\section{Der Patient ist individuell anlässlich eines Gesprächs aufzuklären. Aufklärungsformulare können dies nicht ersetzen.}

Die Aufklärung hat sich inhaltlich nach den dem Impfstoff beiliegenden Fachinformationen zu richten [13]. Ebenso gehört zur Aufklärung, den Patienten über von Gesundheitsbehörden geäusserte Bedenken und Risiken zu informieren.

Weiter ist der Patient darauf hinzuweisen, dass eine Impfung freiwillig ist, sofern dies zutrifft, und ob ein allfälliges Ansteckungsrisiko für Dritte besteht (je nach Impfstoff ist das möglich) [14].

Die Ärztin hat zudem darauf hinzuweisen, ob aufgrund der konkreten Umstände eine Impfung überhaupt notwendig und sinnvoll ist. Das ist aufgrund der Gefährdungslage für jeden einzelnen Patienten im Hinblick auf die Folgen einer Infektion mit dem Erreger, gegen welchen geimpft werden muss, zu prüfen und zu diskutieren. 
Nicht nur über häufig auftretende Risiken muss aufgeklärt werden, sondern auch über seltene, sofern diese bekannt sind und schwere Folgen haben können.

Ebenso ist die Patientin darauf hinzuweisen, dass nicht alle Risiken und Nebenwirkungen bekannt sind, wenn das der Fall ist. Das dürfte vor allem bei Impfstoffen, deren Langzeitfolgen noch unbekannt sind, ein relevantes Thema sein.

\section{Falls noch nicht bekannt ist, ob Langzeitfolgen} möglich sind, so muss der Patient darauf hingewiesen werden.

Die Aufklärung ist zu dokumentieren, damit sie im Falle eines Haftpflichtverfahrens nachvollziehbar ist. Der Patient ist individuell und anlässlich eines Gesprächs aufzuklären. Aufklärungsformulare können allenfalls eine unterstützende Funktion übernehmen, das persönliche Gespräch und die individuelle Aufklärung aber nicht ersetzen. Sie sind entsprechend dem Verlauf des Gesprächs zu ergänzen.

FMH

Sekretariat Rechtsdienst Nussbaumstrasse 29

CH-3000 Bern 15

Tel. 0313591111

Fax 0313591112

lex[at]fmh.ch

Weil sowohl im Falle einer privatrechtlichen Haftung als auch einer Staatshaftung das Verhalten des einzelnen behandelnden Arztes geprüft wird, ist dieser ge- halten sicherzustellen, dass die Behandlung sorgfältig erfolgt. Dazu gehören auch die rechtsgenügende Aufklärung und das Einholen der Einwilligung des Patienten. Sicherstellen kann er das nur, wenn er entweder selbst aufklärt oder weiss, dass die Aufklärung von einem allfälligen, die Impfung organisierenden Gemeinwesen so geplant und durchgeführt wird, dass er dafür nicht haftbar gemacht werden kann.

\section{Literatur}

1 Bundesgesetz über die Produktehaftpflicht (Produktehaftpflichtgesetz, PrHG) vom 18. Juni 1993, SR 221. 112. 944.

2 Art. 2 PrHG

3 Art. 3 PrHG.

4 Art. 4 PrHG.

5 Urteil des Bundesgerichts 4A_365/2014 vom 5. Januar 2015, E. 9.2.

6 Bundesgesetz bettreffend die Ergänzung des Schweizerischen Zivilgesetzbuches (Fünfter Teil: Obligationenrecht, OR) vom 30. März 1911, SR 220, Art. 97ff.

7 Bundesgesetz über die Bekämpfung übertragbarer Krankheiten des Menschen (Epidemiengesetz, EpG) vom 28. September 2012, SR 818.101, Art. 22.

8 Verordnung über die Bekämpfung übertragbarer Krankheiten des Menschen (Epidemienverordnung, EpV) vom 29. April 2015, SR 818.101.1, Art. 38 Abs. 1.

9 Art. 38 Abs. 3 EpV.

10 Art. 56 Abs. 2 EpG; Art. 33 Abs. 2 EpV.

11 Art. 64 EpG.

12 BGE $117 \mathrm{Ib} 197$.

13 Urteil des Bundesgerichts (4A_365/2014) und (4A_371/2014) vom 5. Januar 2015, E. 9.2.
14 BGH VI ZR 189/85 vom 3. Juni 1986; BGH III ZR 52/93 vom 7. Juli 1994 E. II.2a.

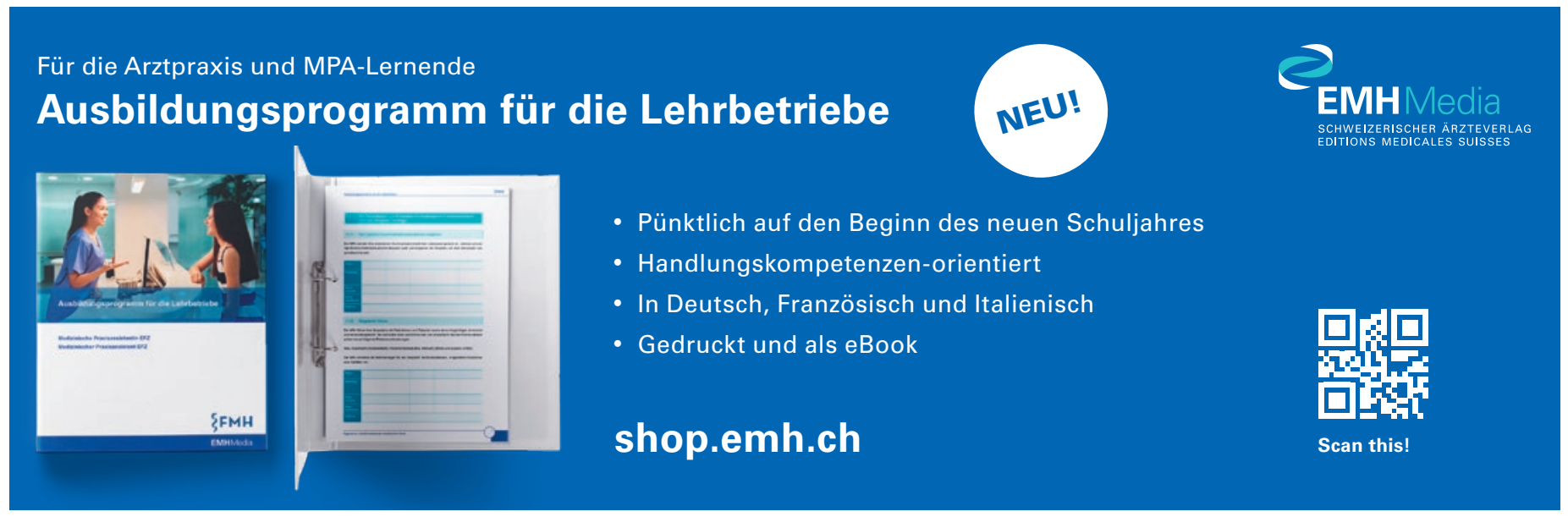

